\title{
Intravenous infusion of 4-AP in chronic spinal cord injured subjects
}

\author{
WH Donovan*,1,4, JA Halter ${ }^{2,4}$, DE Graves ${ }^{2,4}$, AR Blight ${ }^{3}$, O Calvillo ${ }^{2}$, MT McCann ${ }^{2}$, AM Sherwood ${ }^{2,4}$, \\ T Castillo $^{1,4}$, KC Parsons ${ }^{1,4}$ and JR Strayer ${ }^{2,4}$ \\ ${ }^{1}$ The University of Texas Houston Medical School, Houston, TX, USA; ${ }^{2}$ Baylor College of Medicine, Houston, TX, \\ USA; ${ }^{3}$ Acorda Therapeutics, Hawthorne, NY, USA; ${ }^{4}$ The Institute for Rehabilitation and Research, Houston, TX, \\ $U S A$
}

\begin{abstract}
Study design: A prospective double blind cross over trial of intravenous 4-Aminopyridine (4AP).

Objective: To determine the efficacy of this drug in the treatment of spinal cord injured (SCI) patients for neurologic impairment, pain and spasticity.

Setting: The post anesthesia care unit (PACU) of a tertiary care acute hospital.

Methods: Twelve paraplegic patients were enrolled in a double blind cross over intravenous trial of 4-Aminopyridine (4-AP). Thirty milligrams of 4-AP or placebo were administered over a $2 \mathrm{~h}$ period. Patients were serially examined during and after the infusion clinically for pain, sensorimotor function, hypertonicity and motor control using electromyography (EMG). Samples of blood and cerebrospinal fluid (CSF) were also analyzed at similar intervals.

Results: Despite penetration of 4-AP into the CSF, no significant differences were noted in the clinical and EMG parameters at the times measured. Individual changes in sensory function were reported by some patients in both the placebo and 4-AP trials, however mean values were not robust. Frequently, patients complained of unpleasant symptoms during the 4AP infusion.
\end{abstract}

Conclusion: The intravenous route may not be the best way to administer this drug as no short term benefits were observed.

Spinal Cord (2000) 38, 7-15

Keywords: 4-Aminopyridine; spinal cord injury; ASIA impairment scale

\section{Introduction}

Preliminary studies have shown that intravenous administration of 4-Aminopyridine (4-AP) is associated with a range of functional benefits in chronic, incomplete spinal cord injury. These beneficial effects are thought to involve restoration of action potential conduction in damaged, poorly myelinated nerve fibers. 4-AP may also produce a direct enhancement of synaptic transmission. The specific aim of the present study was to examine the neurological and neurophysiological effects of systemic intravenously administered 4-AP in more detail, with a particular emphasis on improved quantification of effects on neurogenic pain, spasticity and residual sensorimotor function in preparation for a subsequent study of intrathecal administered 4-AP.

\section{Background}

Neurological deficits associated with traumatic paraplegia and tetraplegia are attributed to destruction of

*Correspondence: WH Donovan, University of Texas Houston Medical School, Institute for Rehabilitation and Research, Houston, Texas, USA gray matter and the loss of axons in the white matter of the spinal cord as a direct or secondary consequence of physical disruption. In recent years, it has become clear that this simple interpretation is inadequate. A growing body of anatomical evidence from animal models and human injuries indicates that a proportion of the axons often survives, even in severe trauma that results in complete or nearly complete loss of function. ${ }^{1-4}$ Axonal survival may be reflected clinically by a conventional neurologic examination or by responses to reinforcement maneuvers which can reveal latent supraspinal control in muscles that are paralyzed and otherwise thought to be deprived of corticospinal input. ${ }^{5,6}$ It has also been shown that many of the axons that survive at the site of injury can nonetheless be permanently, physiologically disabled, possibly by the combination of demyelination and incomplete remyelination that leaves many of them only thinly myelinated over the longitudinal extent of the lesion. ${ }^{7,8}$

Treatment of demyelinating conditions eg, multiple sclerosis has proven quite difficult but considerable interest has been directed to the drug 4-AP, one of a variety of compounds that blocks potassium channels in nerve membranes. ${ }^{8-14}$ This drug has been shown to 
restore conduction in demyelinated axons under a variety of experimental conditions. In addition to its effect on axonal conduction, 4-AP is also capable of enhancing synaptic transmission by enhancement of presynaptic calcium currents, secondary to potassium channel blockade. A series of experimental trials of 4AP in patients with multiple sclerosis have shown modest improvements in neurological function, effects which are more striking when the subject population is selected on the basis of their response to small changes in temperature. Both intravenous and oral administration of the drug in dogs with chronic, naturally occurring spinal cord injury were shown to produce improvements in motor and sensory function. ${ }^{5,16}$

Based on the results of these studies, several small clinical trials were performed to examine the effects of 4-AP in human spinal cord injury patients. The first, an open label study, examined single intravenous infusions of very low doses in six patients, demonstrated safety and reported minor changes in electrodiagnostic indicators. ${ }^{17}$ The second study, ${ }^{18}$ examined slightly higher doses in a double-blind crossover evaluation of eight patients, and reported beneficial sensorimotor effects in all four of the subjects with incomplete injuries. Quite unexpectedly, this study also indicated that 4-AP provided some relief of chronic central pain and reduction of spasticity in three of the subjects. ${ }^{18}$ This effect on pain was confirmed in the third study, an open trial in six patients, which also demonstrated a remarkable recovery of bowel control and sexual function in one subject. This subject later was given oral 4-AP for a period of 3 weeks with maintenance of the beneficial effects. Prolonged oral administration was subsequently extended to two additional subjects and appeared to produce maintained clinical benefit. ${ }^{19,20}$ A larger, double-blind crossover study of oral, sustained-release 4-AP has now shown more extensive evidence of clinical benefit in incomplete spinal cord injury. ${ }^{16}$

These studies indicate that 4-AP may be useful in the treatment of nervous system conduction deficits in humans. Chronic SCI appears to be particularly amenable to such treatment inasmuch as the anatomical and physiological deficits associated with SCI become stable within the first year or two after injury in most cases. Any beneficial responses to treatment are therefore readily discernible after that period. However, 4-AP does present the potential for serious side effects, given its mechanism of action in blocking potassium channels in neural membranes. At higher doses 4-AP can induce seizures, and is clearly contraindicated in patients with a lowered threshold for seizure activity. The narrow therapeutic index of 4AP restricts the ability to obtain maximal benefit from systemic (oral or intravenous) administration of the drug. If proven safe and effective, 4-AP would constitute the first therapy ever to ameliorate neurological disability in chronic spinal cord injury.

In this study, after Food and Drug Administration and Institutional Review Board approval, 12 patients were recruited to a double-blind crossover analysis studying the effects of intravenous 4-AP compared with vehicle placebo as a basis for a subsequent study of the effects of intrathecal 4-AP infusion. Subjects were studied by a combination of clinical examination, neurophysiological studies and self-report.

\section{Material and methods}

\section{Protocol summary}

After the patient was clinically evaluated and a determination made that the patient was a candidate, the consent forms were signed. Two weeks prior to the first infusion, a baseline measurement of the patient's neurologic and neurophysiologic profile was made by: (1) a standard clinical neurological exam including American Spinal Injury Association (ASIA) motor and sensory scores ${ }^{21}$ (2) a spasticity assessment using the Ashworth scale, ${ }^{22}$ (3) pain questionnaire, ${ }^{23}$ and (4) an evaluation of descending motor control functions using a polyelectromyographic recording technique. ${ }^{24}$ The study designs consisted of a double-blind, placebocontrolled, crossover analysis of intravenous delivery of 4-AP in which each patient was administered the drug and a placebo control on different occasions, separated by 2 weeks. The order of delivery was randomized. The protocol for each subject was the same. The same tests described above were repeated after a baseline prior to infusion of drug or placebo. All members of the research team, save the investigational pharmacists, were blinded to infusion of drug or placebo during the study.

The drug or placebo was then administered for $2 \mathrm{~h}$ through an indwelling venous catheter attached to an infusion pump. Thirty $\mathrm{mg}$ of $4-\mathrm{AP}$ was diluted in $500 \mathrm{ml}$ of dextrose saline and delivered at a dosage of $15 \mathrm{mg} / \mathrm{h}(250 \mathrm{ml} / \mathrm{h})$, up to a maximum of $30 \mathrm{mg}$. Placebo administration consisted of a similar rate of an identically appearing dextrose saline infusion. Infusion was terminated if adverse side effects were noted.

The patient's vital signs were monitored at 30-min intervals throughout the procedure. Samples of blood and urine were obtained from an intravenous catheter in the opposite arm and from the urinary catheter respectively. Cerebrospinal fluid (CSF) was obtained by standard sterile lumbar puncture with or without 22 or 20 ga intrathecal catheter (Burron Accu-bloc Perifix) placement for continuous sampling. All fluid samples were frozen within $30 \mathrm{~min}$ and subsequently transferred to an external laboratory for determination of the level of 4-AP in CSF, blood and urine. Each infusate was also evaluated for drug concentration.

Patients were re-evaluated by each test described above by the same examiner at the end of the period of drug delivery, and then electrodiagnostic tests were repeated. Identical follow-up examinations were then performed at $24 \mathrm{~h}$ after infusion. 


\section{Subject recruitment and selection}

The subjects for this study were recruited from a population of chronic spinal cord injury patients referred to the Institute for Rehabilitation and Research (TIRR) for their rehabilitation care including the management of problems related to pain and/or spasticity. Initial screening and interviews were conducted by the physician co-authors (WH Donovan, KC Parsons, J Strayer). The inclusion criteria were (a) medical diagnosis of chronic ( $>12$ months) paraplegia or paraparesis as a result of traumatic injury; (b) the level of cord injury between T4 and L2; (c) non-ambulatory; (d) medically stable; (e) plain X-ray, MRI, or CT evidence of spinal cord injury at the appropriate level; (f) age between 18 and 55 years, and (g) informed consent. The exclusion criteria were (a) mental incompetence; (b) any untreated infectious disease; (c) previous head injury or brain damage; (d) history of epileptic seizures or lowered seizure threshold; (e) cardiovascular, metabolic, kidney or respiratory abnormalities; (f) known allergy to pyridine containing compounds; (g) currently taking medications for pain and/or spasticity from which they cannot reasonably be discontinued; (h) history of autonomic hyperreflexia, and pregnancy. The characteristics of the patient population and the relative dosing are shown in Table 1.

\section{Clinical measurements}

Pain The subjects' experience of pain was quantified by means of a modified McGill pain questionnaire, which includes 15 descriptions of pain, a grade of severity, and a visual analog scale of pain intensity which asks the patient to grade pain intensity along a continuum of zero to ten. ${ }^{23,25}$ These were used in conjunction with notations regarding hyperesthesia and paresthesias elicited with the sensory examinations along with the patients' reports of subjective unpleasant sensations.

Sensorimotor function Both sensory and motor functions were examined using the ASIA International Standards for Neurologic and Functional Classification of Spinal Cord Injury, revised $1996 .^{21}$ Muscle strength was tested in each of the 20 key muscles, five in each extremity, representing spinal cord segments C5 through $\mathrm{T} 1$ and L2 through S1, employing a six point grading scale $(0-5)$. Since all subjects were paraplegics, with neurologic levels below $\mathrm{T} 1$, all subjects had a baseline motor score of at least 50. Sensory function was tested and scored for two modalities, sharp/dull and light touch in all dermatomes. The former was checked using the sharp and blunt end of a safety pin and the latter was checked using a wisp of cotton. A 3point scale $(0-2)$ was used depending whether the modality was normal (2), diminished (1) or absent (0). All subjects had a baseline sensory score of at least 32 in each modality or 64 for both modalities. Anal motor function and deep anal sensation were also checked on the initial examination for classification of the ASIA Impairment Scale (AIS): A - complete, B - sensory sparing only, extending into the caudal most segments, $\mathrm{C}$ - sensory and motor sparing (more than one half of the key muscles below the neurologic level were $0-2$ ), $\mathrm{D}$ - sensory and motor sparing (at least half of the key muscles below the neurologic level were 3-5), and E full motor and sensory recovery.

Hypertonicity Patients were evaluated for baseline and changes in tone according to two scales, the Ashworth Scale ${ }^{22}$ and the Reflex Scale. ${ }^{26}$ The former grades resistance to passive movement of both hips and knees on a 5-point scale based on increasing difficulty; the latter grades deep tendon reflexes at the knees (patella) and ankles (Achilles) on a 6-point scale based on briskness and clonus.

Table 1 Patient description and injury characteristics

\begin{tabular}{|c|c|c|c|c|c|c|c|c|c|}
\hline \multirow[b]{2}{*}{$I D$} & \multirow[b]{2}{*}{ Gender } & \multirow{2}{*}{\multicolumn{2}{|c|}{$\begin{array}{c}\text { Subject } \\
\text { Weight } \\
(\mathrm{kg})\end{array}$}} & \multirow[b]{2}{*}{$\begin{array}{l}\text { Dose } \\
\text { (mg) }\end{array}$} & \multirow[b]{2}{*}{$m g / K g$} & \multicolumn{4}{|c|}{ Injury } \\
\hline & & & & & & Level & $A I S$ & $\begin{array}{c}\text { Years } \\
\text { post injury }\end{array}$ & Etiology \\
\hline 2 & M & 30 & 93.6 & 30 & 0.320 & $\mathrm{~T}-10$ & A & 3 & GSW \\
\hline 3 & M & 65 & 77.3 & 30 & 0.388 & $\mathrm{~T}-02$ & A & 16 & GSW \\
\hline 10 & M & 45 & 113.6 & 30 & 0.264 & $\mathrm{~T}-05$ & A & 10 & MVA \\
\hline 11 & M & 50 & 84.1 & 30 & 0.357 & $\mathrm{~T}-04$ & A & 29 & MVA \\
\hline 24 & M & 66 & 91.8 & 30 & 0.327 & $\mathrm{~T}-02$ & A & 6 & FALL \\
\hline 4 & M & 61 & 71.3 & 30 & 0.421 & $\mathrm{~T}-09$ & $\mathrm{~B}$ & 27 & FALL \\
\hline 9 & M & 35 & 63.6 & 30 & 0.471 & $\mathrm{~T}-10$ & $\mathrm{C}$ & 7 & FALL \\
\hline 22 & M & 39 & 100.0 & 30 & 0.300 & $\mathrm{~L}-02$ & $\mathrm{C}$ & 13 & MVA \\
\hline 23 & M & 53 & 84.4 & 30 & 0.355 & $\mathrm{~T}-04$ & $\mathrm{C}$ & 6 & FALL \\
\hline 12 & M & 47 & 125.0 & 30 & 0.240 & L-02 & $\mathrm{C}$ & 2 & MVA \\
\hline 17 & M & 42 & 80.0 & 30 & 0.375 & L-02 & $\mathrm{D}$ & 2 & MVA \\
\hline 18 & $\mathrm{~F}$ & 30 & 59.9 & 30 & 0.501 & T-04 & D & 10 & MVA \\
\hline Means & & 46.9 & 87.05 & & 0.360 & & & 10.9 & \\
\hline
\end{tabular}




\section{Electrophysiologic measurements}

Methodology Multichannel surface EMG recordings were used to objectively document motor control and hypertonicity during a standardized sequence of volitional and reflex motor tasks with the subject in a comfortable, supine position, and consisted of a subset of the Brain Motor Control Assessment (BMCA) protocol. ${ }^{24}$ Pairs of recessed, silver-silver chloride surface electrodes were placed $3 \mathrm{~cm}$ apart over the midline of the muscle bellies of the quadriceps, hamstrings, tibialis anterior, triceps surae and flexor hallucis brevis muscles of each lower limb, with additional pairs placed symmetrically about the midline over abdominal muscles (at the level of the umbilicus) and lumbar paraspinal muscles. EMG channels were amplified with a gain of 5000 over a band width of $50-600 \mathrm{~Hz}(-3 \mathrm{db})$, digitized at 1800 samples/s/channel with 12 bit accuracy. After recording the calibration and verifying appropriate electrode placement, data were continuously acquired beginning with relaxation through the end of the protocol (approximately $30 \mathrm{~min}$ ). During drug/placebo infusion, 5-min records of activity during requested relaxation were recorded at half-hour intervals, beginning $30 \mathrm{~min}$ after the infusion started.

In addition to the $12 \mathrm{EMG}$ channels, two additional channels were allocated for level sensors which documented thigh angles, leg angles or foot position. One channel was allocated for transduction of forces exerted during neck flexion and Jendrassik's maneuvers, tendon tap force and plantar stimulation duration and force, selected according to the portion of the protocol. A sixteenth channel was used to record timing of the commands given to the subject, and the maneuvers undertaken. All data were immediately archived onto CD-ROM at the end of the study.

Motor control Recording and Data Reduction: The full bandwidth data was digitized at 1600 samples per second per channel in order to provide an unaliased display of the data (CODAS $^{\mathrm{TM}}$, Dataq, Inc., Akron, $\mathrm{OH}$, USA), but the envelope of activity was used for actual analysis. For this purpose, a root mean square (RMS) algorithm was then applied over successive, 80 point epochs of data, thereby generating the envelope of activity ${ }^{27}$ and compressing the data to an effective sample rate of $20 \mathrm{~s} / \mathrm{s} /$ channel at the same time.

The envelope of the EMG activity $\left(\mathrm{EMG}_{\mathrm{RMS}}\right)$ calculated in terms of the root mean square (RMS) ${ }^{27}$ was used for analysis. For low frequency signals such as transducer outputs, the mean value calculated over the same interval was used rather than a high-pass filtered, RMS value. The envelope data was manually analyzed, beginning with identification and marking of events for each phase of each maneuver. The quantitative representation of motor control used for purposes of spasticity evaluation was based on the RMS voltage recorded from each muscle across sub-phases of each maneuver, extracted from the original record. The extracted segments were used to calculate the average activity for each channel and each of the segments in $\mu \mathrm{V}_{\text {RMS }}$. Fixed, 8-s segments were taken for reinforcement maneuvers and 5-s segments for voluntary, passive and plantar stimulation maneuvers.

These average values were then entered into a spreadsheet for subsequent manipulation. Data from repeated studies were evaluated in terms of ratios, ${ }^{28}$ in order to obtain a dimensionless value which may be summarized or compared across muscles and subjects. In all cases, identical maneuvers collected under identical conditions were compared. Ratios were calculated from baseline-corrected data separately for each muscle and each phase of each maneuver, with the minimum level set to $0.1 \mu \mathrm{V}$ to avoid a division by zero in the ratio calculation (ibid). Using group means and standard deviations from previous studies of SCI subjects (ibid), standard scores were calculated for each subject, comparing the three post-infusion times to the baseline recording. ${ }^{24}$

\section{Analysis methods}

The data from this investigation were analyzed using multivariate repeated measures analysis of variance for the ASIA motor and sensory scales and the Visual Analog Scale (VAS). Data from the Ashworth scale was analyzed using the Friedman's test for $k$ related samples, a nonparametric repeated measures test. All analyses were conducted with an $a$ priori alpha of .05. All analyses were performed with SPSS 8.0.

\section{ASIA scales}

The design for this analysis was one within subjects (four levels) and two between subjects factors (two levels each). The within subjects factor was the total motor, pin prick and light touch scores as dependent variables for the measurements taken at baseline, 2.5, 4 and $24 \mathrm{~h}$ post infusion. The between subject factors were the active or placebo condition and a grouping of the AIS score for the patient, (A\&B vs C\&D).

\section{McGill visual analogue scale}

The Visual Analog Pain Scale was also analyzed with multivariate repeated measures analysis of variance. The design for this analysis was one within subjects factor (three levels) and one between subjects factor (two levels each). Patients completed the VAS at baseline, 4 and $24 \mathrm{~h}$ post infusion.

\section{$B M C A$}

The reduced data from the Hip and Knee Flexion and Extension portion of the BMCA studies in standard score form were analyzed using repeated measures ANOVA. Standard scores from measurements from 
2.5, 4 and $24 \mathrm{~h}$ post infusion were analyzed as dependent variables in a one within (time) and one between (medication) subject factor design. ${ }^{28}$

\section{Analysis of 4-AP in plasma and CSF}

Plasma samples were obtained from the subjects at the following intervals: at baseline, after insertion of the intravenous catheter, but prior to 4-AP administration (through a separate catheter), and then at 1, 2, 2.5, 3, 4,8 and $24 \mathrm{~h}$ after the beginning of infusion of the drug. Each sample consisted of approximately $7 \mathrm{~mL}$ of blood collected into a $10 \mathrm{~mL}$, preservative free, heparinized tube. Samples were centrifuged within $30 \mathrm{~min}$ of collection, where possible, and no later than $60 \mathrm{~min}$ from collection. The resulting plasma was frozen at less than $-20^{\circ} \mathrm{C}$ for storage and shipment to the analysis laboratory. 4-AP concentrations in the plasma samples were measured using a previously validated HPLC techniques, by PHARMout Laboratories, Sunnyvale, CA, USA. The method has a quantification limit of $2.00 \mathrm{ng} / \mathrm{ml}$ for 4-aminopyridine, using $1 \mathrm{~mL}$ of plasma for analysis. Accuracy and precision of analyses were monitored by concurrent analysis of samples of normal plasma (from subjects outside the study) spiked with known quantities of 4-AP.

Samples of CSF were also obtained during and after intravenous infusion of 4-AP by means of an intrathecal catheter, inserted from a lumbar puncture and threaded as close to the site of injury to the spinal cord as possible, using fluoroscopic visualization. These fluid samples were obtained at times equivalent to those of the plasma samples, and were centrifuged and frozen in a similar manner for storage and shipment. The analytical procedures were similar to those used for the plasma, but were separately validated, and similarly monitored with spiked samples of plain CSF. The quantification limit for 4-AP was $2 \mathrm{ng} / \mathrm{ml}$, using $0.5 \mathrm{~mL}$ of CSF for analysis.

\section{Results}

Strict adherence to the protocol was generally accomplished as intended. However, two studies had to be repeated, one due to the subject's not adhering to the required constant level of anti-spasticity medications, and one to an error in the infusion ie, the same ingredient was repeated. Thus a total of 26 studies were run.

The most obvious difference between the placebo and active branches was the discomfort experienced by the subjects at the infusion site, leading to a change in dilution (to half strength) after the second trial. Two subjects experienced so much discomfort that they requested that infusion be halted before the full quantity was infused. One remained quite agitated for several hours, making it necessary to skip one of the evaluation sets.

\section{Laboratory results}

This study demonstrates that 4-AP administered intravenously does reach doses of 30 to $80 \mathrm{ng} / \mathrm{ml}$ (mean 55) at the end of a $2 \mathrm{~h}$ infusion when given according to the methods mentioned, ie $15 \mathrm{mg}$ per hour IV over $2 \mathrm{~h}$. Similarly, 4-AP was shown to enter the $\mathrm{CSF}$, reaching a mean peak of $25 \mathrm{ng} / \mathrm{ml} 1 \mathrm{~h}$ after the cessation of the infusion, but actually a level close to this was maintained from the end of the infusion until $2 \mathrm{~h}$ later. Six hours later it still measured $15 \mathrm{ng}$ $\mathrm{ml}$ in both serum and CSF but was gone by $24 \mathrm{~h}$. A full description of the pharmacokinetic data will be presented separately.

\section{Clinical observations}

- (A) Motor scores When the motor scores were compared at the designated times pre- and postinfusion between the 4-AP and placebo, no significant changes were noted at any of the three points post-infusion nor between the two groups (Figure 1). When the motor incomplete patients $(n=6)$ were similarly compared with the motor complete patients when both received 4-AP, no differences in motor response were detected. No differences were also found when the motor complete and incomplete groups were separately compared with placebo.

- (B) Sensory scores The scores for both sensory modalities, pinprick and light touch, were similarly analyzed, comparing the 4-AP trials $v s$ placebo and comparing the motor complete group vs the motor incomplete group receiving 4-AP (Figures 2 and 3), no significant differences were found between any of the scores

- (C) Visual Analog Scale (VAS) When the 4-AP trials were compared to the placebo, the difference in VAS reached significance $(P<0.05)$. The 4-AP group had more pain at $4 \mathrm{~h}$ and less at $24 \mathrm{~h}$ (Figure 4). When the motor incomplete group was

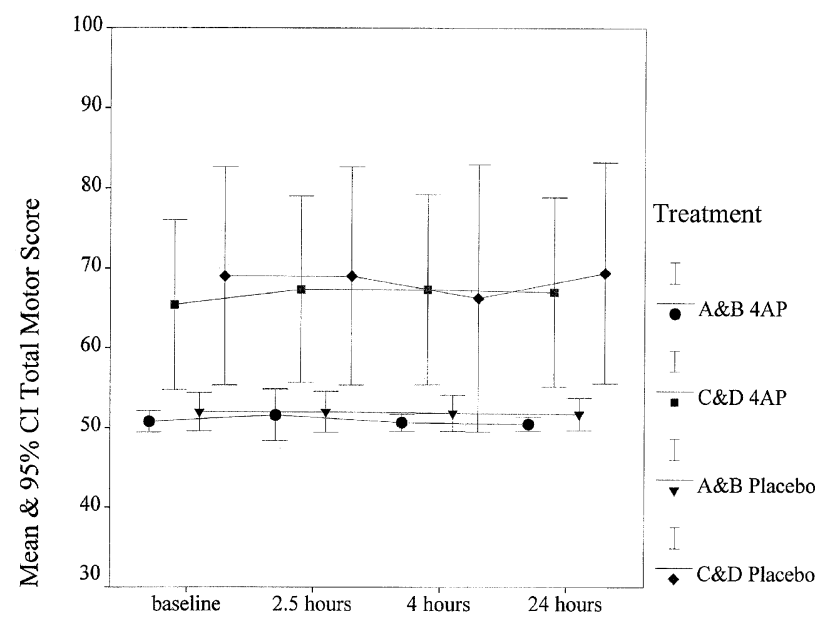

Figure 1 ASIA motor scores 


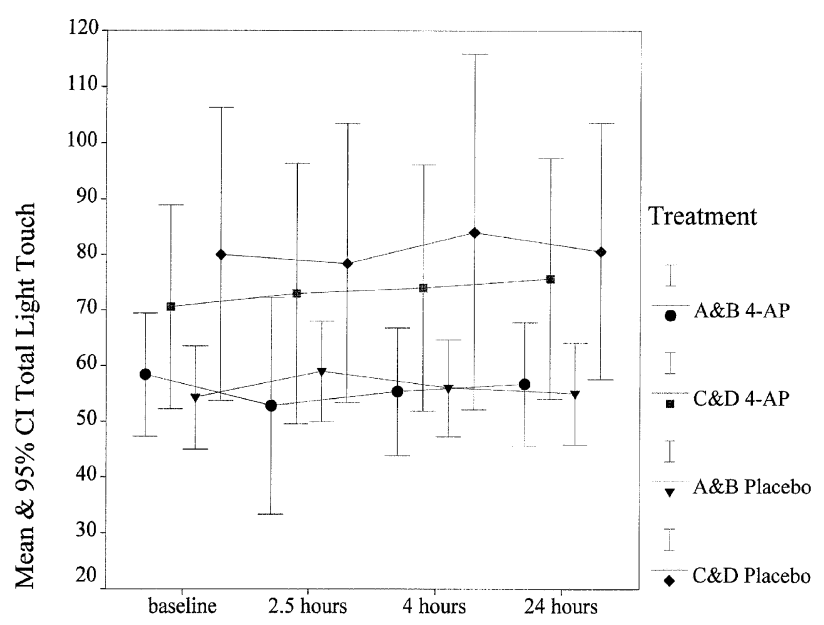

Figure 2 ASIA Light Touch scores

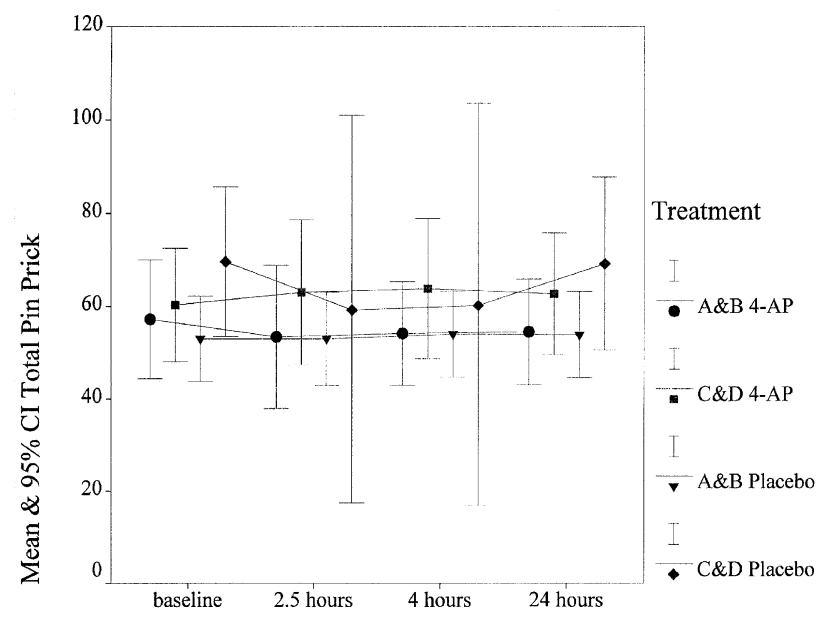

Figure 3 ASIA Pin Prick scores

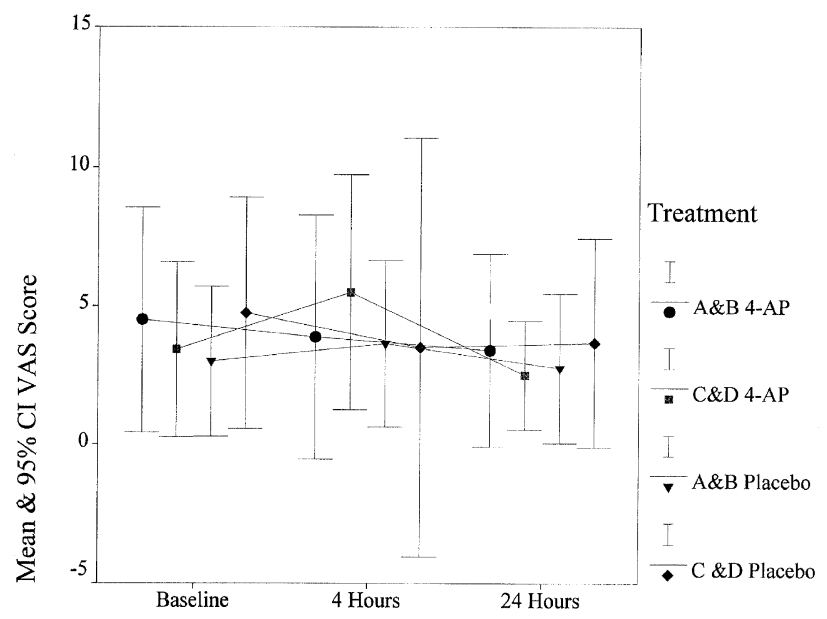

Figure 4 McGill Visual Analog Scale compared to the motor complete group when both received 4-AP, no difference was noted.

The changes in scores between baseline and at the end of the infusion of placebo and 4-AP for each patient are shown in Table 2. Patient number 12 for example experienced a loss of one muscle grade in one muscle and a gain of one muscle grade in another after the placebo infusion. She also noted a decrease in score of 1 in eight dermatomes and an increase in score of 2 in two dermatomes and an increase in score of 1 in nine dermatomes for a total of 13. She noted no motor change after the 4-AP infusion and did not participate in the sensory evaluation because of too much discomfort at that time.

- (D) The descriptions of the type of pain experienced by the subjects at $2 \mathrm{~h}$ (at the end of the infusion) and $24 \mathrm{~h}$ after the start of the infusion are shown in Table 3.

- (E) The Ashworth scores and the reflex scores are shown in Table 4 . There were no significant changes in the placebo or treatment groups over time.

- (F) Patients were asked to report their symptoms during and $4 \mathrm{~h}$ after the infusion of 4-AP. The symptoms were grouped into five categories and are shown in Table 5. Clearly different degrees of symptoms between the two trials were reported in almost all categories.

\section{Neurophysiological studies of motor control: BMCA} results

Of all the neurophysiologic measurements, the only data that were clearly analyzable, ie free of background noise, were the EMG activity in response to passive movement of the hips and knees. This is shown in Figure 5. The differences in the placebo and 4-AP trials did not reach significance. None of the results of the BMCA were robust enough to reach statistical significance when evaluated across all 12 subjects. This is not surprising when one realizes that many of the individual subjects showed no substantial effect at all, and the effects which were present were not consistent across the subjects.

\section{Discussion}

In general, the protocol was executed as planned. A drawback to the manner in which the study was conducted was the fact that the subjects were all outpatients; they came to the post anesthesia care unit early on the morning of the trial. Thus, the pre-trial conditions were not well controlled. Several subjects were not local and had to spend the night in a local hotel before the study, others came from home. These factors may have contributed to instability in baseline conditions between trials. 
Clinical

Analysis of the CSF, blood and urine samples after unblinding revealed that the 4-AP and placebo were successfully infused in the manner prescribed in the protocol. Plasma levels peaked at about $2 \mathrm{~h}$, i.e. at the end of the infusion. CSF values reached a peak $30-$ 60 min later. No measurable physiologic effects were noted at these levels, however, patients did report symptomatic changes. The amount of drug infused was slightly less than has been used in animal studies that have indicated various improvements in neurological function. ${ }^{15}$ Such studies have typically used bolus injections of $0.5-1 \mathrm{mg} / \mathrm{kg} 4-\mathrm{AP}$, which may produce significantly higher peak plasma and CSF levels.

Table 2 Changes individual ASIA scale items for patients during 4-AP and placebo trials

\begin{tabular}{|c|c|c|c|c|c|c|c|c|}
\hline \multirow{2}{*}{$I D$} & \multicolumn{4}{|c|}{$\begin{array}{c}\text { Placebo } \\
\text { Change in score }\end{array}$} & \multicolumn{4}{|c|}{$\begin{array}{c}\text { 4- } A P \\
\text { Change in score }\end{array}$} \\
\hline & $-v e$ & $+v e$ & $-y \rho$ & $+v e$ & $-v e$ & $+v e$ & - ve & + vo \\
\hline 2 & 1 & 1 & 0 & 1 & 0 & 0 & 0 & 0 \\
\hline 3 & 0 & 1 & 6 & 5 & 0 & 0 & 10 & 3 \\
\hline 10 & 0 & 0 & 2 & 5 & 0 & 0 & - & - \\
\hline 11 & 0 & 0 & 2 & 0 & 0 & 0 & 7 & 1 \\
\hline 24 & 0 & 0 & 3 & 3 & 0 & 0 & 2 & 2 \\
\hline 4 & 0 & 0 & 1 & 1 & 0 & 0 & 1 & 0 \\
\hline 9 & 0 & 1 & 0 & 2 & 0 & 0 & 3 & 2 \\
\hline 22 & 0 & 0 & 10 & 7 & 0 & 0 & 2 & 2 \\
\hline 23 & 0 & 0 & 2 & 5 & 0 & 0 & 0 & 1 \\
\hline 12 & 0 & 0 & 7 & 3 & 0 & 0 & 8 & 7 \\
\hline 17 & 1 & 0 & 8 & 4 & 0 & 0 & 6 & 10 \\
\hline 18 & 1 & 1 & 8 & 13 & 0 & 0 & - & - \\
\hline Total & 3 & 4 & 49 & 49 & 0 & 0 & 39 & 28 \\
\hline
\end{tabular}

Table 3 Patient description of pain during study

\begin{tabular}{|c|c|c|c|c|c|c|}
\hline & Baseline & $\begin{array}{c}4-A P \\
\text { Time } \\
4 h\end{array}$ & $24 h$ & Base line & $\begin{array}{c}\text { Placebo } \\
\text { Time } \\
4 h\end{array}$ & $24 h$ \\
\hline Throbbing & 0.91 & 0.86 & 0.50 & 1.00 & 1.00 & 0.82 \\
\hline Soothing & 0.50 & 0.57 & 0.50 & 0.73 & 0.67 & 0.45 \\
\hline Stabbing & 0.91 & 0.57 & 0.44 & 0.78 & 0.63 & 0.50 \\
\hline Sharp & 0.64 & 1.00 & 0.44 & 1.00 & 0.75 & 0.50 \\
\hline Cramping & 0.80 & 0.57 & 0.63 & 1.00 & 0.89 & 0.60 \\
\hline Gnawing & 0.91 & 0.71 & 0.50 & 1.10 & 0.78 & 0.70 \\
\hline Hot-Burning & 1.64 & 1.78 & 1.00 & 1.55 & 1.11 & 1.17 \\
\hline Aching & 1.00 & 1.25 & 0.70 & 1.27 & 1.13 & 1.00 \\
\hline Heavy & 0.90 & 0.75 & 0.56 & 0.67 & 1.00 & 1.00 \\
\hline Tender & 0.60 & 0.57 & 0.56 & 0.67 & 0.63 & 0.40 \\
\hline Splitting & 0.40 & 0.83 & 0.50 & 0.56 & 0.75 & 0.40 \\
\hline Tiring-Exhausting & 1.10 & 1.40 & 0.75 & 1.11 & 1.25 & 0.80 \\
\hline Sickening & 0.60 & 0.71 & 0.56 & 0.67 & 0.88 & 0.60 \\
\hline Punishing-Cruel & 0.89 & 0.50 & 0.63 & 0.89 & 0.88 & 0.50 \\
\hline
\end{tabular}

Table 4 Mean Ashworth and Reflex scores for the 4-AP and placebo trials

\begin{tabular}{|c|c|c|c|c|c|c|c|c|}
\hline & \multicolumn{2}{|c|}{$\begin{array}{c}\text { Baseline } \\
\text { Type }\end{array}$} & \multicolumn{2}{|c|}{$\begin{array}{l}2.5 h \\
\text { Type }\end{array}$} & \multicolumn{2}{|c|}{$\begin{array}{c}4 h \\
\text { Type }\end{array}$} & \multicolumn{2}{|c|}{$\begin{array}{l}24 h \\
\text { Type }\end{array}$} \\
\hline & $4-A P$ & Placebo & $4-A P$ & Placebo & 4- $A P$ & Placebo & 4- $A P$ & Placebo \\
\hline Ashworth Score Right & 2.58 & 2.58 & 2.92 & 2.92 & 2.50 & 2.82 & 2.83 & 2.75 \\
\hline Ashworth Score Left & 2.42 & 2.50 & 2.92 & 3.00 & 2.58 & 2.82 & 2.50 & 2.82 \\
\hline Reflex Score Right & 1.58 & 2.00 & 1.58 & 2.17 & 1.75 & 2.36 & 1.42 & 2.17 \\
\hline Reflex Score Left & 1.83 & 2.00 & 2.08 & 2.00 & 2.08 & 2.09 & 1.58 & 2.17 \\
\hline
\end{tabular}


Table 5 Adverse reactions during and after infusion

\begin{tabular}{lcccc}
\hline & \multicolumn{2}{c}{ Infusion } & \multicolumn{2}{c}{ Post infusion } \\
& Placebo & $4-$ AP & Placebo & 4-AP \\
\hline Local pain and numbness & 0 & 9 & 0 & 0 \\
Dysesthesias & 1 & 9 & 0 & 3 \\
Involuntary contractions & 3 & 4 & 0 & 4 \\
Vascular dysfunction & 1 & 8 & 1 & 9 \\
Abdominal distress & 0 & 4 & 0 & 4 \\
\hline
\end{tabular}

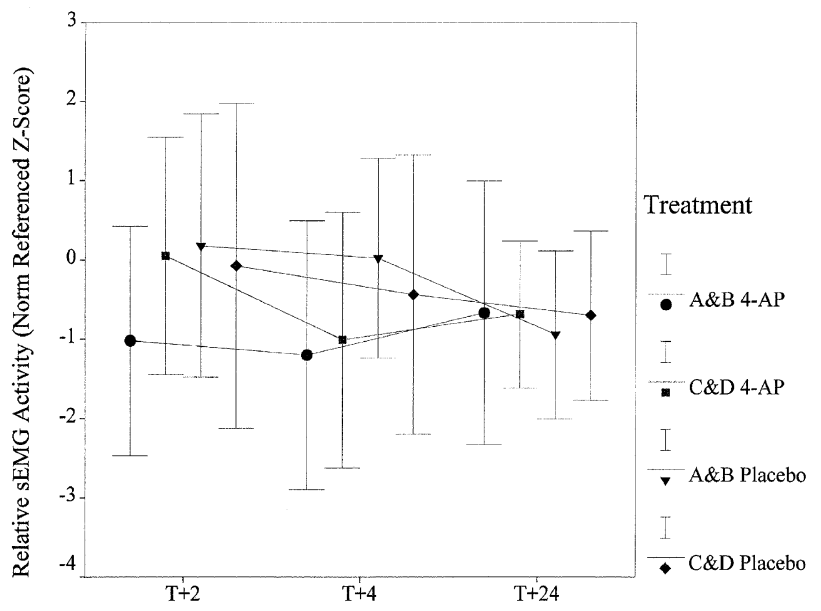

Figure 5 Passive Hip and Knee Flexion

However, plasma and CSF levels have not been monitored in an equivalent way in animal studies of functional changes, so it is not possible to make a direct comparison at this time.

Mean scores stayed remarkably constant across the time line, although this was against a background of considerable variability, particularly in neurological grades $\mathrm{C}$ and $\mathrm{D}$. The range of sensory scores varied over time, and this was somewhat more noticeable during the placebo infusions. Sensory scores have been shown to have less intra-rater reliability ${ }^{29}$ than motor scores, and the variability found in the sensory scores pre- and post-infusion supports this. It should be noted that a previous study, using a similar doubleblind crossover design with intravenous injection of this drug also did not demonstrate significant changes in mean values of motor or sensory function with small numbers of subjects. ${ }^{18}$ In that study, there were individual subjects that showed marked neurological changes with the drug but not the placebo. A doubleblind crossover study with a larger number of subjects and more prolonged administration of an oral formulation of the $\operatorname{drug}^{16}$ did report statistically significant neurological improvement using responder-analysis. However, even in that larger study, it was not possible to demonstrate significant differences between the measures of sensory and motor function used in the present study, given the underlying variability and a limited number of subjects. Therefore, the apparently negative outcome of this study may not be inconsistent with previous findings, given its intrinic limitations.

Although the differences between the placebo and treatment trials did reach significance in the VAS, the authors feel that this was probably due to the greater amount of overall pain reported by subjects during the 4-AP infusions. Most reported local pain at the infusion site and could not distinguish between neurogenic pain and their overall discomfort. Surprisingly the resistance to passive movement was essentially unchanged both clinically and neurophysiologically throughout the time line despite the increase in discomfort reported by most patients during the 4-AP infusion.

\section{Neurophysiological}

Results from the BMCA evaluation were not striking; however, the consistency of the studies during many of the trials suggest that the protocol was able to document motor status of the subjects. Conversely, there was a relatively poor consistency in baseline levels, indicating that the way in which the protocol was conducted may have contributed to the lack of effects. In some instances, there was a noticeable increase in activity during the drug infusion indicating a greater sensitivity than the Ashworth scale. This increase in tone may have been due to a direct effect on overall excitability, or may have been due to a secondary effect due to subjects' discomfort with drug infusion.

The general lack of robustness in any of the results may be due to an inadequate level of 4-AP, an insufficient time of administration, the specific pathophysiology of the subjects selected, or the inability of the drug to alter motor control in such subjects (lesions in the spinal cord below the cervical segment).

\section{Conclusion}

The intravenous infusion of 4-AP did not appear to alter measurements of any of the parameters tested, except pain, despite its penetration into the CSF. Because no short term effects were seen and because of frequent complaints of pain during the infusion, the IV route may not be the best way to conduct evaluations of this drug.

\section{Acknowledgments}

This project was supported in part by a grant from the Kent Waldrep National Paralysis Foundation. 


\section{References}

1 Blight AR. Cellular morphology of chronic spinal cord injury in the cat: analysis of myelinated axons by line-sampling. Neuroscience 1983; 10: $521-543$.

2 Blight AR, DeCrescito V. Morphometric analysis of experimental spinal cord injury in the cat: the relation of injury intensity to survival of myelinated axons. Neuroscience 1986; 19: $321-341$.

3 Kakulas BA. Pathology of spinal injuries. Cent Nerv Syst Trauma 1984; 1: $117-129$.

4 Bunge RP et al. Observations on the pathology of human spinal cord injury. In Seil, FJ (ed): Advances in Neurology, Vol. 59. Neural Injury and Regeneration. New York: Raven Press, 1993; pp. $75-89$.

5 Dimitrijevic MR, Dimitrijevic MM, Faganel J, Sherwood AM. Suprasegmentally induced motor unit activity in paralyzed muscles of patients with established spinal cord injury. Ann Neurol 1984; 16: 216-221.

6 Hayes KC et al. Reinforcement of motor evoked potentials in patients with spinal cord injury. In Levy WJ, Cracco RQ, Barker AT, Rothwell J (eds) Magnetic Motor Stimulation: Based Principles and Clinical Experience (EEG Supplement 43). Amsterdam: Elsevier Science Publishers, BV, 1991; pp 312-329.

7 Blight AR. Axonal physiology of chronic spinal cord injury in the cat: intracellular recording in vitro. Neuroscience 1983; 10: 1471 1486.

8 Blight AR. Effect of 4-aminopyridine on axonal conductionblock in chronic spinal cord injury. Brain Res Bulletin 1989; 22: $47-52$.

9 Jones RE et al. Effects of 4-aminopyridine in patients with multiple sclerosis. J Neurol Sci 1983; 60: $353-362$.

10 Stefoski D, Davis FA, Faut M, Schauf CL. 4-Aminopyridine improves clinical signs in multiple sclerosis. Ann Neurol 1987; 21: $71-77$.

11 Stefoski D et al. 4-Aminopyridine in multiple sclerosis: Prolonged administration. Neurology 1991; 41: $1344-1348$.

12 van Diemen $\mathrm{H}$ et al. The Effect of 4-Aminopyridine on Clinical Signs in Multiple Sclerosis: A Randomized, Placebo-Controlled, Double-Blind, Cross-over Study. Ann Neurol 1992; 32: 123-130.

13 van Diemen $\mathrm{H}$ et al. 4-aminopyridine induces functional improvement in multiple sclerosis patients: A neurophysiological study. J Neurol Sci 1993; 116: 220 -226.
14 Bever Jr C. The current status of studies of aminopyridines in patients with multiple sclerosis. Ann Neurol 1994; 36: S118S121.

15 Blight AR, Toombs JP, Bauer MS, Widmer WR. The effects of 4 aminopyridine on neurological deficits in chronic cases of traumatic spinal cord injury in dogs: a phase I clinical trial. $J$ Neurotrauma 1991; 8: $103-119$.

16 Potter PJ et al. Randomized double-blind Crossover trial of Tampridine-SR (sustained release 4-Aminopyridine) in patients with incomplete spinal cord injury. J Neurotrauma 1998; 15: $837-849$.

17 Hayes $\mathrm{KC}$ et al. Preclinical trial of 4-aminopyridine in patients with chronic spinal cord injury. Paraplegia 1993; 31: 216-224.

18 Hansebout RR, Blight AR, Fawcett S, Reddy K. 4-aminopyridine in chronic spinal cord injury: a controlled, double-blind, crossover study in eight patients. J Neurotrauma 1993; 10: $1-18$

19 Hayes KC et al. 4-Aminopyridine-sensitive neurologic deficits in patients with spinal cord injury. J Neurotrauma 1994; 11: 433 446

20 Hayes $\mathrm{KC}$ et al. Effects of oral 4-aminopyridine on neurological function in patients with spinal cord injury. J Neurotrauma 1995; 12: 495.

21 ASIA: International Standard for Neurological and Functional Classification of Spinal Cord Injury, Chicago, American Spinal Injury Association, 1996

22 Ashworth B. Preliminary trial of carisoprodol in multiple sclerosis. Practitioner 1964; 192: 540 - 542.

23 Melzack R. The McGill pain questionnaire: major properties and scoring methods. Pain 1975; 1: 277-299.

24 Sherwood AM, McKay WB, Dimitrijevic MR. Motor control after spinal cord injury: assessment using surface EMG. Muscle Nerve 1996; 19: 966 - 979 .

25 Wegener ST, Elliott TR. Pain assessment in spinal cord injury. Clin J Pain 1992; 8: 93 - 101.

26 Penn RD et al. Intrathecal baclofen for severe spinal spasticity. $N$ Engl J Med 1989; 320: $1517-1521$.

27 Basmajian JV, De Luca CJ. Muscles Alive: Their Functions Revealed by Electromyography, Fifth Edition, Baltimore, Williams and Wilkins, 1985.

28 Sherwood AM, Priebe MM, Graves DE Consistency of multichannel surface EMG recordings: application in spinal cord injured subjects. J Electrophysiol Kinesiol 1997; 7: $97-111$.

29 Clifton GL et al. Omental transposition in chronic spinal cord injury. Paraplegia 1996; 34: $193-203$. 